$\infty$

0

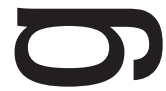

-

ת

1

$\longrightarrow$ 



\title{
ANÁLISE DAS PRECIPITAÇÕES MENSAIS DA BACIA HIDROGRÁFICA DO RIO BRIGIDA NO SEMI-ÁRIDO PERNAMBUCANO, USANDO O MÉTODO DE WARDS
}

\author{
ANÁLISE DAS PRECIPITAÇÕES MENSAIS DA BACIA HIDROGRÁFICA DO RIO BRÍGIDA NO SEMI- \\ ÁRIDO PERNAMBUCANO, USANDO O MÉTODO DE WARDS
}

\author{
José Alegnoberto Leite Fechine - UFPE \\ fechini02@hotmail.com.br \\ Josicleda Domiciano Galvíncio - UFPE \\ josicleda@ibest.com.br
}

\section{RESUMO}

0 objetivo deste trabalho foi 0 de determinar regiões pluviometricamente homogêneas no âmbito da bacia hidrográfica do rio Brígida, utilizando dados de precipitação mensal de seis estações meteorológicas, com séries amostrais de 29 anos, 1964 a 2006. Os critérios de seleção dos postos foram: que as séries não tivessem falhas e que os anos fossem concomitantes. Com esses critérios foram selecionados os municípios de Parnamirim, Moreilândia, Trindade, Ipubi, Exu, Ouricuri, Araripina, Serrita e Bodocó, localizados na bacia do rio Brígida, semi-árido do estado de Pernambuco. Utilizou-se no estudo o método hierárquico aglomerativo de Ward, tendo como medida de dissimilaridade a distância euclidiana. Os municípios de Moreilândia, Serrita, Parnamirim, Araripina e Bodocó se agruparam e assim possuem quantitativos de índices pluviométricos similares. Esta comprovação mostrou-se necessária para garantir a aderência das análises, onde se objetivou identificar perfis de relacionamento de precipitação entre os municípios analisados.

Palavras-chave: climatologia, análise de agrupamento, Ward e semi-árido

\section{ABSTRACT}

The purpose of this study was to determine rainfall homogeneous regions within the basin of the river Brígida, using data from monthly rainfall of six meteorological stations, with series sample of 29 years, 1964 to 2006. The selection criteria for the posts were not that the series had flaws and that the years were concurrent. With these criteria were selected municipalities of Melbourn, Moreilândia, Trinidad, IPUB, Eshu, Spalding, Ayr, Serrita and Bodocó, located in the basin of the river Brígida, semiarid state of Pernambuco. It was used in the study method hierarchical cluster of Ward, with the measure of dissimilarity the Euclidean distance. The municipalities of Moreilândia, Serrita, Melbourn, Ayr and Bodocó have formed a group and thus have quantitative indices of similar rainfall. This evidence proved to be necessary to ensure the adherence of the analysis, which is aimed to identify profiles of relationship between the municipalities of precipitation analyzed.

Key-words: climatology, analysis of grouping, Ward, semi-arid

\begin{tabular}{|l|c|c|c|c|c|c|}
\hline Boletim Goiano de Geografia & Goiânia - Goiás - Brasil & v. 28 & n. 2 & p. 75-88 & jul. / dez. & 2008 \\
\hline
\end{tabular}





\section{Introdução}

Estudar os recursos hídricos de uma bacia hidrográfica requer o conhecimento das características climáticas da bacia. Muitas das vezes essas informações não existem e quando existem não são regionais e sim pontuais (estações meteorológicas). O estudo climatológico de uma determinada região sem levar em consideração um único ponto de observação (estação meteorológica), consiste em conhecer as características de determinada área, envolvendo dados de outros pontos. Esses pontos, que representam regiões, podem ser obtidos através de técnicas estatísticas apropriadas. A técnica mais utilizada, em diversas áreas do conhecimento científico, é a análise multivariada, através dela podemos obter grupos homogêneos de variáveis em questão e investigar o seu comportamento espacial e temporal, (Araújo, 2005). Esse comportamento espacial e temporal da climatologia de uma bacia hidrográfica e de suma importância para a modelagem hidrológica distribuída.

Atualmente, existem na literatura vários métodos de agrupamento associados às várias funções de agrupamento, gerando diferentes formas de grupos. Os relacionados por Wilks (1995) são: Método da Ligação Simples, Método da Ligação Completa, Método do Centróide e Método de Ward.

Com o objetivo de determinar regiões homogêneas através de técnicas estatísticas como: Análise de Componentes Principais e Análise de Agrupamento, utilizando como variável de agrupamento dados de precipitação e como método de agrupamento o de Ward, muitos trabalhos já foram realizados. Baptista da Silva et al., (2001) utilizando análise de agrupamento estudaram o comportamento dos totais trimestrais das chuvas no estado do Ceará, a partir de 20 estações meteorológicas distribuídas por toda extensão do Estado, tendo como medida de similaridade a distância euclidiana ao quadrado e a inércia intraclasse como critério de agregação.

Souza et al. (1992) fizeram a homogeneização da precipitação pluvial para o estado de Alagoas, utilizando análise de agrupamento com o objetivo de avaliar o comportamento das precipitações pluviais médias e máximas. No estudo os autores fizeram uso de dados de 60 estações, com um período mínimo de 14 e um máximo de 72 anos. Silva et al. (1996) determinaram grupos homogêneos de precipitação no setor leste da região Nordeste. No estudo utilizaram dados totais mensais de precipitação de 127 postos meteorológicos localizados nos tabuleiros costeiros e baixadas litorâneas de municípios dos estados do Ceará, Rio Grande do Norte, Paraíba, 
Pernambuco, Alagoas, Sergipe e Bahia. Os dados foram selecionados através dos critérios de concomitância entre os anos e entre estados e que a série fosse superior a 20 anos. Os grupos foram obtidos através do método de agrupamento de Ward.

Lopes et al. (1996) através do método da ligação completa e do método da ligação simples obtiveram cinco grupos pluviometricamente homogêneos no estado do Rio Grande do Norte. Araújo (2005) apud Rodrigues et al. (1996) usaram o método da classificação hierárquica na identificação de regiões pluviometricamente homogêneas no estado do Ceará. Seis grupos de regiões pluviometricamente homogêneas foram obtidos para o estado. As regiões homogêneas, obtidas da análise de agrupamento, são usadas para vários fins como, por exemplo, classificação climatológica, (Becker, 1992).

Galvíncio et al. (2001) usaram análise de agrupamento, através do critério de ligação simples para determinar microrregiões pluviometricamente homogêneas no estado da Paraíba. Neste estudo os autores analisaram o agrupamento dos municípios que possuía os mesmos quantitativos climáticos para o mês de março, o mês mais chuvoso do semi-árido do estado da Paraíba. O método se mostrou muito eficiente na formação dos grupos.

O objetivo deste trabalho foi o de determinar regiões pluviometricamente homogêneas no âmbito da bacia hidrográfica do rio Brígida.

Os resultados deste estudo darão suporte a modelos hidrológicos distribuídos sobre a bacia hidrográfica do rio Brígida.

\section{Material e métodos}

A pesquisa consistiu em determinar o grau de aglomeração entre os dados de precipitação dos municípios. Para tanto, utilizou-se análise de correlações, calculando-se a distância euclidiana para determinar o nível de agrupamento existente entre as variáveis.

O escopo do estudo realizado partiu da seleção de uma parte significante dos dados de precipitação da bacia hidrográfica do rio Brígida entre os anos de 1964 a 2006. Os critérios de seleção dos postos foram que: as séries não tivessem falhas e que os anos fossem concomitantes. Com esses critérios foram selecionados os municípios de Parnamirim, Moreilândia, Trindade, Ipubi, Exu, Ouricuri, Araripina, Serrita e Bodocó, localizados na bacia do rio Brígida, semi-árido do estado de Pernambuco, Figura 1. 


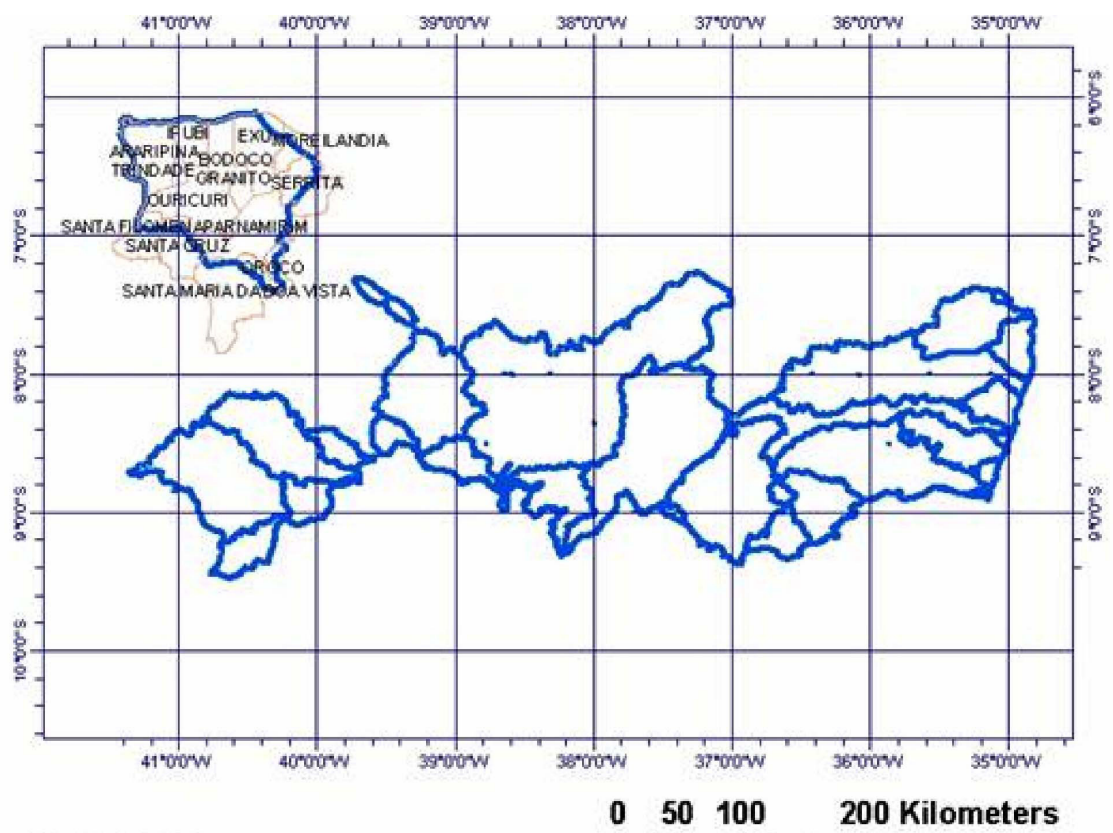

LEGENDA

Bacias do Estado de Pernambuco

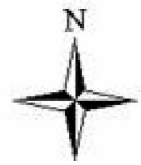

Figura 1 - Estado de Pernambuco com suas respectivas bacias hidrográficas e a bacia do Brígida com seus municípios

Como seguimento do trabalho, buscou-se identificar padrões de agrupamentos entre os municípios analisados, através de um padrão de comportamento e / ou características comuns que expliquem a relação entre as precipitações. Para tanto, foi utilizado o método de aglomeração conhecido por Aglomeração Hierárquica (Hierarquical Cluster). Particularmente, foi utilizado o método de Ward, que é um método de variância, derivado de um processo hierárquico e aglomerativo. O método de Ward tem por objetivo minimizar o quadrado da distância euclidiana às médias dos conglomerados. A distância euclidiana é a raiz quadrada da soma dos quadrados das diferenças de valores para cada variável. Este é um dos métodos mais utilizados em estudos de cluster e mostrou-se adequado às características das variáveis em consideração. 
O método de Ward forma grupos, minimizando a dissimilaridade, ou minimizando o total das somas de quadrados dentro de grupos, também conhecida como soma de quadrados dos desvios (SQD). Em cada etapa do procedimento, são formados grupos, de tal maneira que a solução resultante tenha o menor SQD dentro de grupos. Nessas etapas, são consideradas as uniões de todos os possíveis pares de grupos e, os dois que resultam em menor aumento de SQD são agrupados até que todos os grupos formem um único, reunindo todos os indivíduos (Everitt \& Dunn, 1991).

\section{Resultados e discussão}

A Tabela 1 apresenta as combinações geradas através do método de agrupamento de Ward. Pode-se observar que os municípios analisados formam três conglomerados distintos Moreilândia, Serrita e Parnamirim, Araripina e Bodocó. Nos quais Trindade e Exu apresenta perfil diferenciado dos demais. O coeficiente mostra a distância de aglomeração entre os municípios de Parnamirim e Trindade que é de 4913,233 - estágio 8 - combinação dos dados de 1 - 3, aqui também se encontra incluído o município de Exu. O estágio 7 - Parnamirim e Ouricuri, o coeficiente é de 4125,472, combinação dos dados 1 - 6, e é bem menor do que o coeficiente do estágio 8. Ou seja, a precipitação das cidades de Ipubi, Ouricuri, Trindade e Exu ultrapassam as precipitações das cidades de Moreilândia, Serrita, Parnamirim, Araripina e Bodocó. Ainda de acordo com os resultados de aglomeração, Figura 2, os municípios que melhor se agrupam são os do estágio 1 - combinação dos dados 2 e 8 e o coeficiente da inércia de 455,420, representando os municípios de Moreilândia e Serrita - pode-se incluir aqui o município de Parnamirim, que possui coeficiente de aglomeração bem aproximo. No estágio 2 - combinação dos dados 7 e 9, o coeficiente foi de 950,604, representando os municípios de Araripina e Bodocó. Desta forma, os municípios de Moreilândia, Serrita, Parnamirim, Araripina e Bodocó são os que possuem as variações de chuva mais semelhantes (variação de precipitação mensal). Já os municípios de Trindade e Exu são os que mais se distanciam, estágio 6 - combinação 3 e 5, coeficiente de 3379,532, desta forma podemos colocar em um grupo separado Tabela 1 
Tabela 1 - Resultados da análise de aglomerados.

\begin{tabular}{l|l|l|l|l|l|l}
\hline \multirow{2}{*}{ Estágio } & \multicolumn{2}{|c|}{ Combinação dos dados } & Coeficiente & Estágio de aglomeração de dados & $\begin{array}{c}\text { Próximo } \\
\text { Estágio }\end{array}$ \\
\hline & Dados 1 & Dados 2 & & Dados 1 & Dados 2 & \\
\hline 1 & 2 - Moreilândia & $8-$ Serrita & 455,420 & 0 & 0 & 3 \\
\hline 2 & 7 - Araripina & 9 - Bodocó & 950,604 & 0 & 0 & 4 \\
\hline 3 & 1 - Parnamirim & - -Moreilândia & 1463,011 & 0 & 1 & 4 \\
\hline 4 & 1 - Parnamirim & 7 - Araripina & 2051,258 & 3 & 2 & 5 \\
\hline 5 & 1 - Parnamirim & 4 - Ipubi & 2709,112 & 4 & 0 & 7 \\
\hline 6 & 3 - Trindade & 5 - Exu & 3379,532 & 0 & 0 & 8 \\
\hline 7 & 1 - Parnamirim & 6 - Ouricuri & 4125,472 & 5 & 0 & 8 \\
\hline 8 & 1 - Parnamirim & 3 - Trindade & 4913,233 & 7 & 6 & 0 \\
\hline
\end{tabular}

A Figura 2 apresenta o dendrograma de opções de agrupamento de precipitação da bacia hidrográfica do rio Brígida. As linhas verticais representam os conglomerados e as linhas horizontais a distância euclidiana entre os mesmos. A escolha do número de regiões no dendrograma foi feita através da analise dos agrupamentos, obtendo-se três regiões pluviometricamente homogêneas no âmbito da bacia hidrográfica do rio Brígida, Figura 3. A partir da análise dos conglomerados criados, as seguintes aglomerações de municípios foram identificadas os seguintes grupos:

- Grupo I: Moreilândia, Serrita e Parnamirim;

- Grupo I: Araripina e Bodocó;

- Grupo II: Ipubi;

- Grupo III: Ouricuri;

- Grupo IV: Trindade e Exu

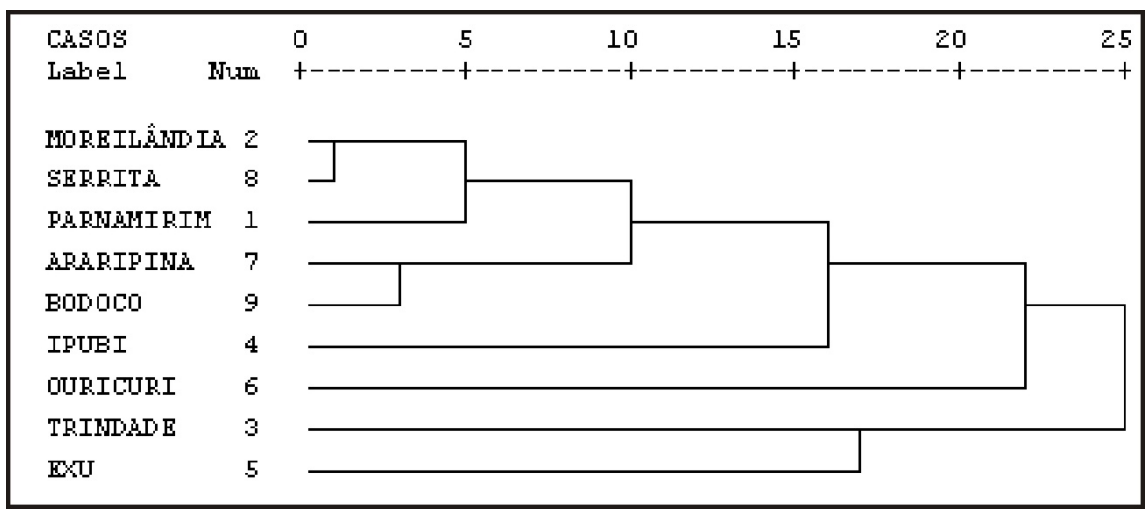

Figura 2 - Dendrograma 


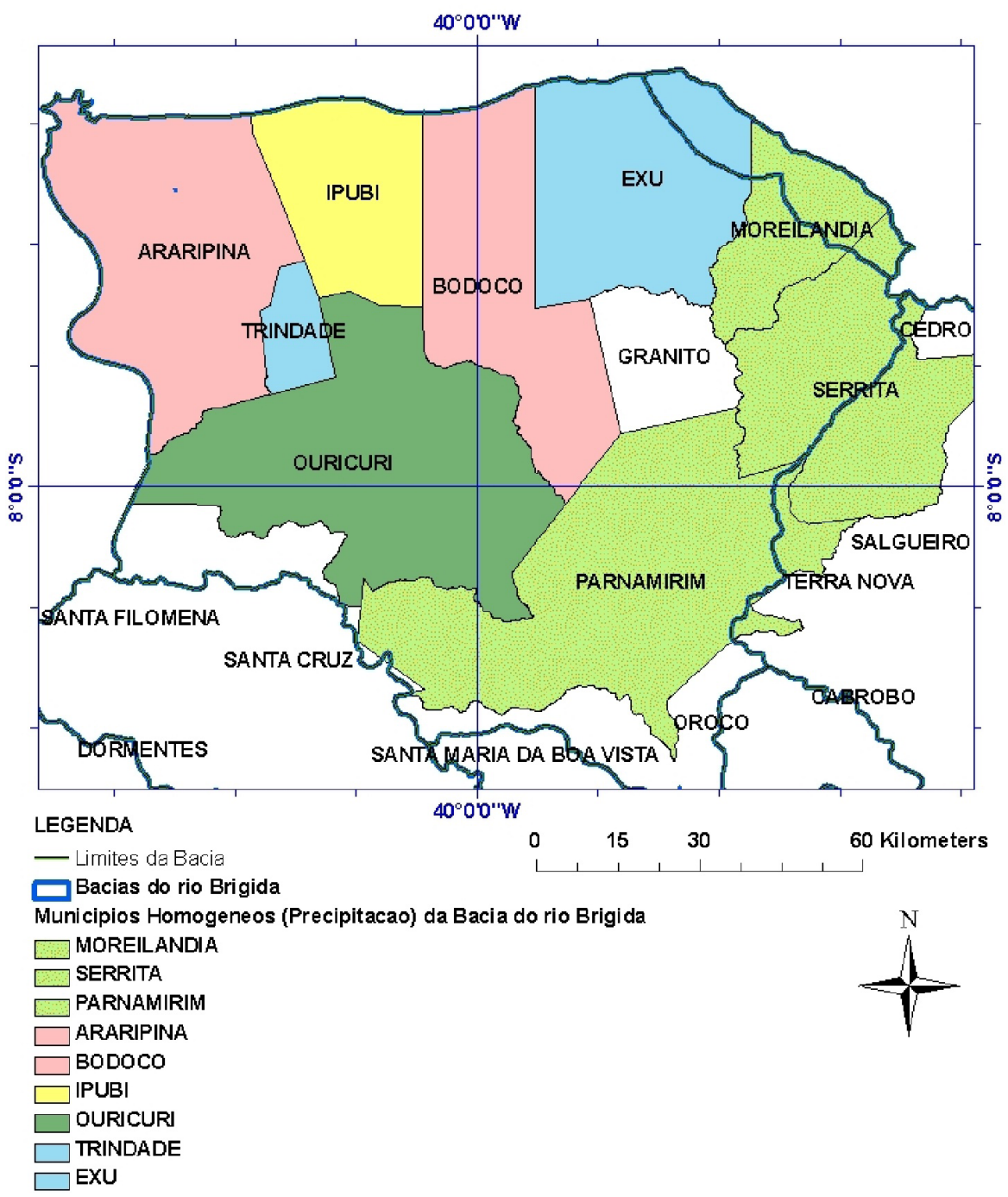

Figura 3 - Regionalização da precipitação mensal na bacia do rio Brígida, através do método de Análise de Agrupamento e critério de Ward.

Os municípios de Exu e Trindade são o que mais se distancia dos demais municípios. O município de Parnamirim se agrupa no grupo I, porém ele está próximo da média de precipitação quando considerado a média dos outros municípios do Grupo I, como mostra a Figura 4. 
Dos pontos analisados o município de Exu e Trindade é o que mais contribui em termo de altura pluviométrica para a "alimentação" da bacia do rio Brígida.

O município de Araripina apresenta média de precipitação bastante próxima da dos municípios de Exu, Trindade e Bodocó, 63, 62, 47 e $53 \mathrm{~mm}$, Figura 4. Isso mostra a importância de agrupar as precipitações com totais mensais e não com médias mensais. Portanto, a média tende a mascarar a realidade das variações pluviométricas da bacia em estudo. De fato, apesar da existência de boa proximidade da média de precipitação, esse município, se caracteriza como municípios de perfil diferente, linha vermelha da Figura 4

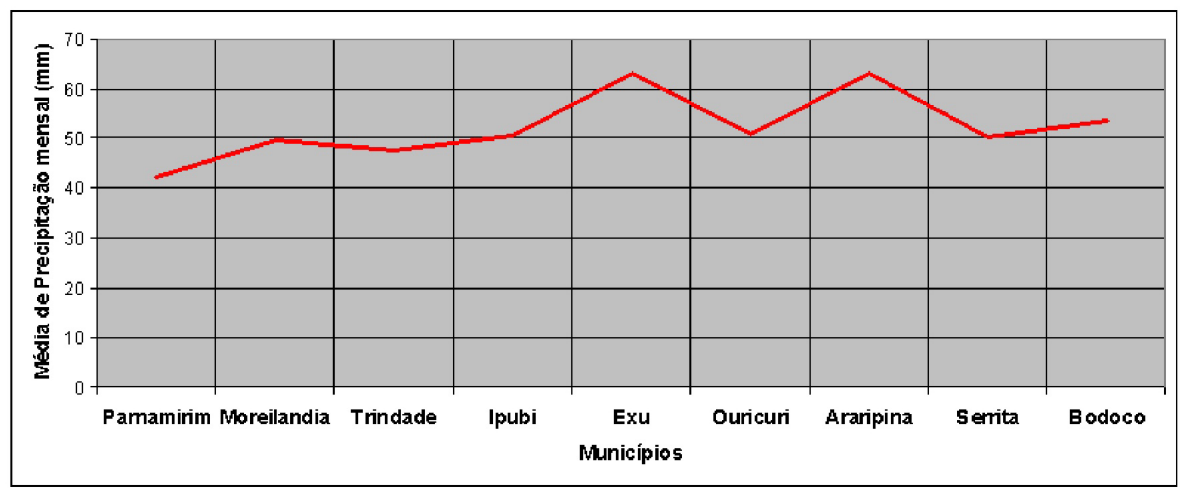

Figura 4 - Variação da precipitação mensal no âmbito da bacia hidrográfica do rio Brígida

Além das semelhanças das variações pluviométricas mensais dos municípios de Moreilândia e Serrita ainda se assemelham com os seus valores médios, como apresentado na Figura 5. Levando em consideração apenas a média mensal de precipitação o agrupamento seria como apresentado na Figura 5. Ou seja, aconteceria uma alteração na regionalização da precipitação, o que torna o método não é aconselhado homogeneizar precipitações. Se assim fosse, os municípios de Moreilândia, Serrita, Trindade, Ouricuri e Ipubi, teriam as mesmas precipitações, "regiões homogêneas"; isto também aconteceria com os municípios de Exu e Araripina. Já Bodocó e Parnamirim ficariam em grupos isolados. 


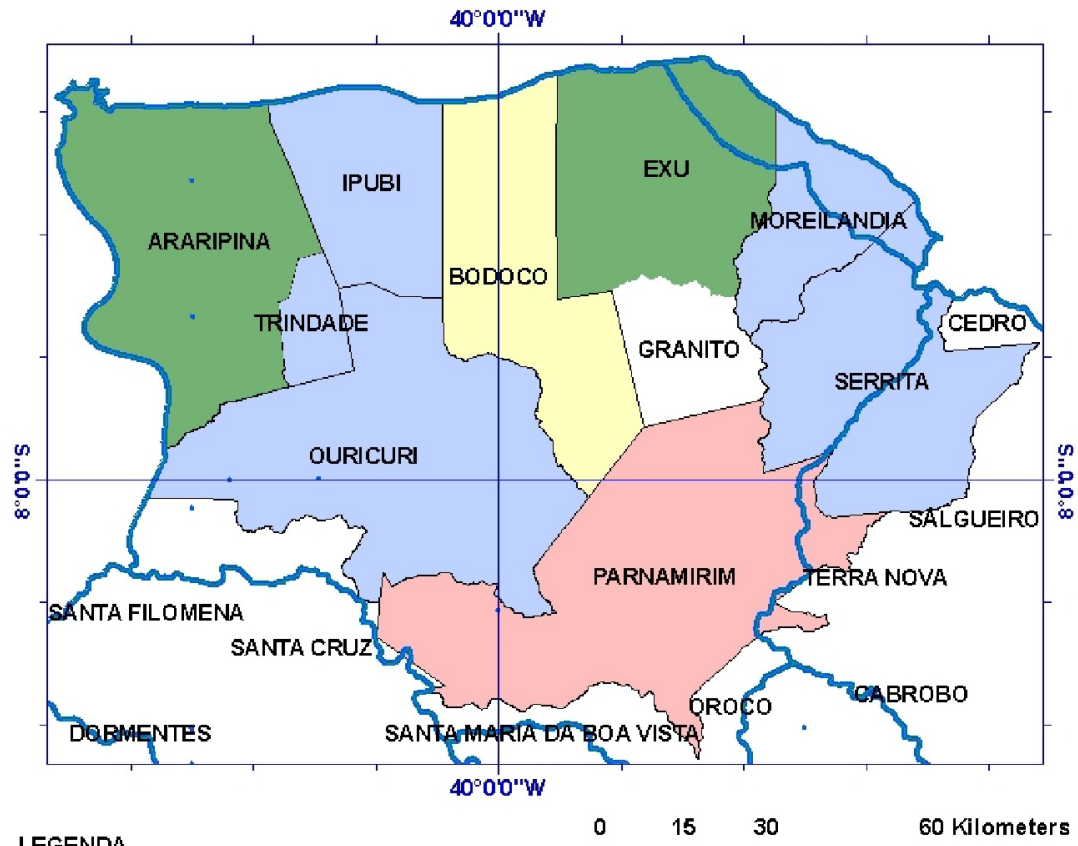

LEGENDA

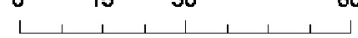

Killometers

- Limites da Bacia
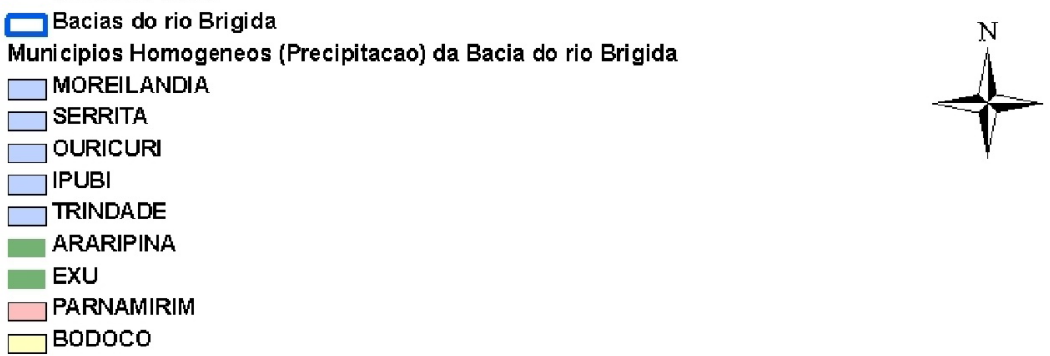

Figura 5 - Regionalização da precipitação da bacia hidrográfica do rio Brígida em função da média pluviométrica.

Neste estudo as médias mensais serviram para validar o método, pois a média tende a mascarar a realidade das variações pluviométricas da bacia em estudo.

Com base nos resultados encontrados buscou-se identificar as principais características e similaridades observadas diretamente nos dados observados nos municípios. Enfim, de acordo com os conglomerados, método de 
agrupamento de Wards, os municípios que melhor se agrupam são: Moreilândia, Serrita, Parnamirim, Araripina e Bodocó.

\section{Conclusões}

Conclui-se que a bacia hidrográfica do rio Brígida pode ser regionalizada em três grupos pluviometricamentes homogêneos. Os municípios que possuem características climáticas mais homogêneas são: Moreilândia, Serrita, Parnamirm, Araripina e Bodocó. Sendo que, Parnamirim também se assemelha as características climáticas desses municípios, mas não tão fortemente como os supracitados. Exu e Trindade não se assemelham as variações climáticas destes municípios da bacia hidrográfica do rio Brígida. Exu e Trindade se destacam com as maiores precipitações da bacia, indicando assim o seu potencial de contribuição para o escoamento superficial da bacia hidrográfica do rio Brígida.

\section{Referências}

ARAÚJO, S. M. B. Estudo da variabilidade climática em regiões homogêneas de temperaturas médias do ar no Rio Grande do Sul. Dissertação de mestrado em Meteorologia. Universidade Federal de Pelotas, 29/05/2005.

ASSIS, F.N. de ARRUDA, H.V. de PEREIRA, A.R. Aplicações de estatística a climatologia: teoria e prática. Pelotas: Universitária, 1996.

BAPTISTA DA SILVA, J. et al. Modelagem das chuvas trimestrais por regióes homogêneas no Estado do Ceará. Revista Brasileira de Agrometeorologia, Santa Maria, V.09, n. 02. p. 317-324, 2001.

BECKER, C. T. Determinação de regiões homogêneas de precipitação e temperatura no estado do Rio Grande do Sul. Campina Grande: UFPB, 1992. 80 f. Dissertação (Mestrado em Meteorologia - Climatologia) - Curso de Pós-graduação em Meteorologia, Universidade Federal da Paraíba, Campina Grande, 1992.

BUNCHAFT, G.; KELLNER, S. Estatística sem mistérios. V 2. 2ª ed. Petrópolis: Vozes, 1999.

DOWNING, D., CLARK, J. Estatística Aplicada. São Paulo: Saraiva, 2a . Ed. 2002.

EVERITT, B.S.; DUNN, G. Applied multivariate analysis. London: Edward Arnold, 1991.

EVERITT, B. Cluster Analysis. London: Heinemann Books, 1974.

FOVELL, R. G.; FOVELL, M. C. Climate Zones of the Conterminous United States Defined Using Custer Analysis. Jounal of Climate, Boston, v.6, n.11, p. 2103-2135, 1993. 
GADGIL, S.; IYENGAR, R. N. Cluster analysis of rainfall stations of the Indian peninsula. Quarterly Journal of the Royal Meteorological Society, London, v.106, n.512, p. 873-886, 1980.

GALVÍNCIO, J. D. ; MOURA, Magna Soelma Beserra de ; PRESTE, A. C. A. ; AL, E. . Determinações de Microrregiões Pluviometricamente Homogêneas do Estado da Paraíba. In: 520 Reunião da SBPC, 2000, Brasília, 2000.

HERRERA, O. M. et al. Agrupamento de estações climatológicas localizadas no estado de São Paulo, Brasil, utilizando-se análise multivariada: comparação com classificação de Koppen. In: CONGRESSO ARGENTINO DE METEOROLOGIA, 7.; CONGRESSO LATINAMERICANO E IBÉRICO DE METEOROLOGIA, 7., 1996, Buenos Aires. Anais... Buenos Aires: Biblioteca Nacional, 1996. p. 233-234.

KIM, I. S. et al. Regionalização do estado do Paraná com utilização dos campos de precipitação, temperatura máxima e mínima. In: CONGRESSO BRASILEIRO DE METEOROLOGIA, 11., 2000, Rio de Janeiro. Anais... Rio de Janeiro: SBMet, 2000. CL-000869. 1 CD-ROM.

LEVINE M. David, BERSON L. Mark, STEPHAN David. Estatística: Teoria e Aplicações. Rio de Janeiro: LTC, 2000.

LOPES, P. M. O. et al. Identificação de grupos pluviometricamente homegêneos no estado do Rio Grande do Norte. In: CONGRESSO BRASILEIRO DE METEOROLOGIA, 9., 1996, Campos do Jordão. Anais... Rio de Janeiro: SBMet, 1996. p. 272-274.

RAMOS, M. C. Divisive and hierarchical clustering techniques to analyse variability of rainfall distribution patterns in a Mediterranean region. Atmospheric Research, New York, v.57, p. 123-138, 2001.

RICHMAN, M. B.; LAMB, P. J. Climatic Pattern Analysis of Three-and Seven - Day Summer Rainfall in the Central United States: Some Methodological Considerations and a Regionalization. Jounal of Climate and Applied Meteorology, Boston, v.24, n.12, p. 1325-1343, 1985.

RODRIGUES, M. F. G. et al. Uso da classificação hierárquica na identificação de regiões pluviometricamente homogêneas no estado do Ceará. In: CONGRESSO BRASILEIRO DE METEOROLOGIA, 9., 1996, Campos do Jordão. Anais... Rio de Janeiro: SBMet, 1996. p. 275-280.

SILVA, A. G. et al. Análise de agrupamento aplicada ao regime pluviométrico da costa leste e norte do nordeste do Brasil. In: CONGRESSO BRASILEIRO DE METEOROLOGIA, 9., 1996, Campos do Jordão. Anais.... Rio de Janeiro: SBMet, 1996. p. 231-235.

SOUZA, J. L. de. et al. Agrupamento de estações pluviométrica do estado de Alagoas, utilizando-se análise multivariada. Revista Brasileira de Meteorologia, Rio de Janeiro,v.7, n.2, p.603612, 1992.

WILKS, D. S. Statistical Methods in the Atmospheric Sciences. San Diego - CA: Academic Press, 1995.

WOLTER, K. The Southern Oscillation in Surface Circulation and Climate over the Tropical Atlantic, Eastern Pacific, and Indian Oceans as Captured by Custer Analysis. Jounal of Climate and Applied Meteorology, Boston, v.26, n.12, p. 540-558, 1987.

YU, T. Y.; CHANG, L. F. W. Delineation of air-quality basins utilizing multivariate statistical methods in Taiwan. Atmospheric Environment, New York, v.35, n 18, p.3155-3166, 2001. 
28, n. 2: 73-86, 2008

Artigo

$\left.87\right|_{\infty} ^{:}$

José Alegnoberto Leite Fechine - Doutorado em Geografia na Universidade Federal de Pernambuco

Josicleda Domiciano Galvíncio - Professor adjunto da Universidade Federal de Pernambuco, Chefe do Departamento de Ciências Geográficas

Recebido para publicação em outubro de 2008

Aceito para publicação em dezembro de 2008 
